Asian Spine Journal Vol. 6, No. 3, pp 203 206, 2012

http://dx.doi.org/10.4184/asj.2012.6.3.203

\title{
Solitary Epidural Lipoma with Ipsilateral Facet Arthritis Causing Lumbar Radiculopathy
}

\author{
Hong Kyun Kim¹, Sung Hye Koh², Kook Jin Chung ${ }^{1}$ \\ 1'Department of Orthopaedic Surgery, Kangnam Sacred Heart Hospital, Hallym University College of Medicine, Seoul, Korea \\ ${ }^{2}$ Department of Radiology, Hallym Univerisy Sacred Heart Hospital, Hallym University College of Medicine, Anyang, Korea
}

A 55-year-old obese man (body mass index, $31.6 \mathrm{~kg} / \mathrm{m}^{2}$ ) presented radiating pain and motor weakness in the left leg. Magnetic resonance imaging showed an epidural mass posterior to the L5 vertebral body, which was isosignal to subcutaneous fat and it asymmetrically compressed the left side of the cauda equina and the exiting left L5 nerve root on the axial T1 weighted images. Severe arthritis of the left facet joint and edema of the bone marrow regarding the left pedicle were also found. As far as we know, there have been no reports concerning a solitary epidural lipoma combined with ipsilateral facet arthorsis causing lumbar radiculopathy. Solitary epidural lipoma with ipsilateral facet arthritis causing lumbar radiculopathy was removed after the failure of conservative treatment. After decompression, the neurologic deficit was relieved. At a 2 year follow-up, motor weakness had completely recovered and the patient was satisfied with the result. We recommend that a solitary epidural lipoma causing neurologic deficit should be excised at the time of diagnosis.

Key Words: Solitary epidural lipoma, Posterior facet, Ipsilateral arthritis, Lumbar radiculopathy

\section{Introduction}

Spinal epidural lipomatosis (SEL) is defined as normal adipose tissue that is pathologically overgrown in the epidural space of the spinal canal. It usually occurs in obese people, patients with history of corticosteroid use or endocrinopathy $[1,2]$. Thoracic spine is a site with common predilection and it may extend over several levels in this area. It can cause myelopathy in the thoracic spine or neurogenic intermittent claudication in the lumbosacral area. Solitary spinal epidural lipoma is exceedingly rare. We herein report a rare case of solitary epidural lipoma causing lumbar radiculopathy associated with unilateral arthritis of the posterior facet joint unresponsive to conservative treatment.

\section{Case Report}

A 55-year-old man presented with pain radiating down the left leg. He had no history of corticosteroid use, endocrinopathy and radicular pain. On examination, he was obese (height $165 \mathrm{~cm}$, weight $86 \mathrm{~kg}$ ) with a body mass index (BMI) of $31.6 \mathrm{~kg} / \mathrm{m}^{2}$. He experienced severe limitation in straight leg raise test and motor weakness of the left extensor hallucis longus and peroneal muscles (fair), although sensation was intact. Plain radiographs showed no evidence of abnormality, including instability and other pathologic findings. Magnetic resonance imaging (MRI) revealed an epidural mass posterior to the $\mathrm{L} 5$ vertebral body that was isosignal to subcutaneous fat, and it compressed asymmetrically on the left side of the cauda equina and the exiting left L5 nerve root on sagittal (Fig. 1) and axial (Fig. 2) T1 weighted images (T1WI). Severe arthritis of the left facet joint and edema of the left pedicle bone marrow were also demonstrated (Fig. 3). There was no evidence of spinal stenosis. The result of electromyography was compatible with radiculopathy in the left fifth lumbar root. Leg pain (visual analogue scale [VAS]

Received Mar 21, 2011; Revised Apr 26, 2011; Accepted Apr 27, 2011

Corresponding author: Kook Jin Chung, MD

Department of Orthopaedic Surgery, Kangnam Sacred Heart Hospital, Hallym University College of Medicine,

1 Singil-ro, Yeongdeungpo-gu, Seoul 150-950, Korea

Tel: +82-2-829-5165, Fax: +82-2-834-1728, E-mail: chungkjmd@dreamwiz.com

Copyright (C) 2012 by Korean Society of Spine Surgery

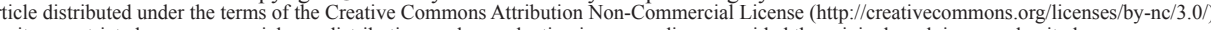
which permits unrestricted non-commercial use, distribution, and reproduction in any medium, provided the original work is properly cited. Asian Spine Journal • pISSN 1976-1902 eISSN 1976-7846 
9) did not respond to physiotherapy and opioid analgesia. We recommended surgical intervention to relieve pain and reverse neurologic deficit, but the patient declined. Selective nerve root block (SNRB) was subsequently performed and his symptom became much improved, and was discharged with pain relief (VAS 3) and neurologic improvement (normal) following two further sessions of SNRB. His symptoms recurred three months after discharge, but pain and motor weakness (good) were not relieved by repeat SNRB. He underwent mass extirpation through left hemilaminot- omy at the level of L5. Intraoperatively, a well-demarcated encapsulated mass measuring $0.9 \times 0.9 \times 2.0 \mathrm{~cm}$ was found in the ventral side of the cauda equina exiting the left L5 nerve root. Left nerve root at the level of L5 was severely swollen due to mass effect. The operation was uneventful and pathologic examination confirmed the excised mass to be a solitary epidural lipoma (Fig. 4). Two years postoperation, parasthesia remained without associated motor weakness. He was satisfied with the surgical result.
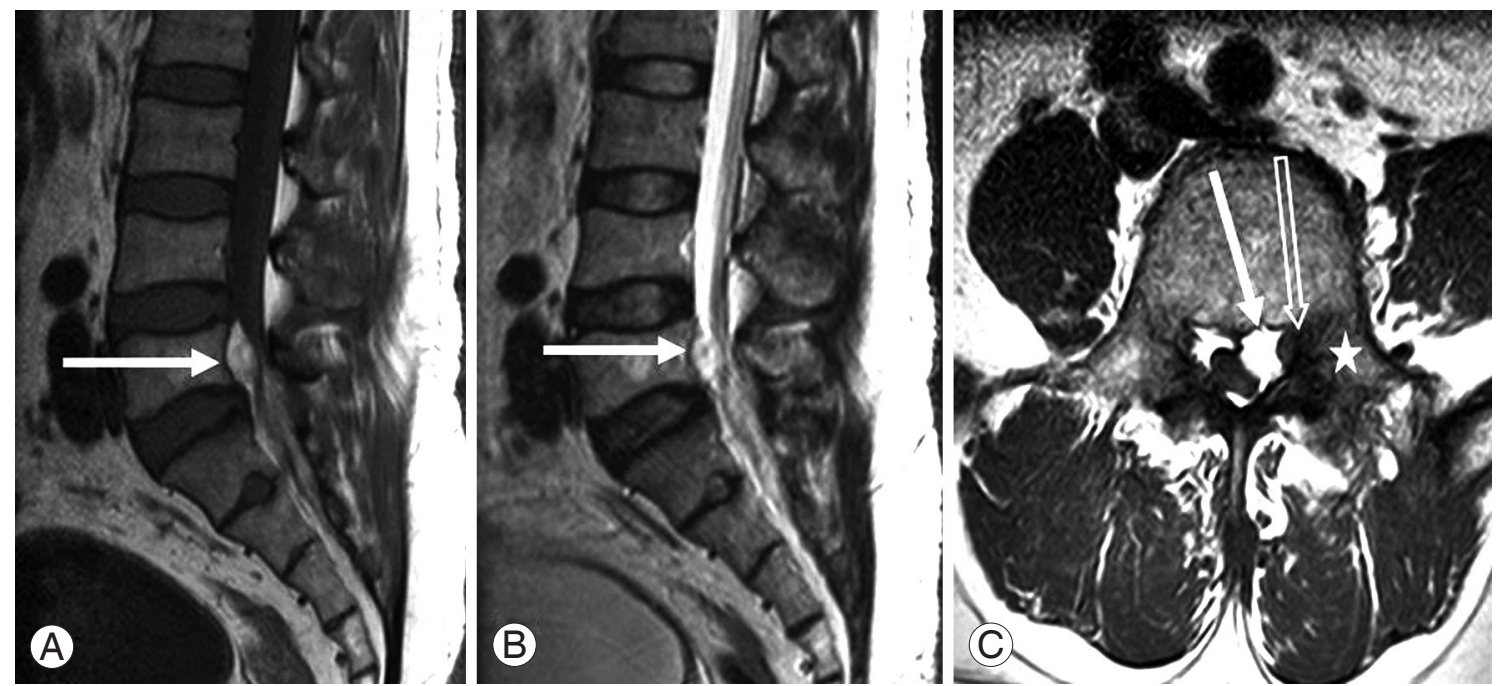

Fig. 1. T1-weighted (A) and T2-weighted (B) magnetic resonance (MR) sagittal images of the left paracentral area showing a mass (arrow) in the spinal canal at L5. The mass is of high signal intensity on both T1-weighted (A) and T2-weighted scans (B). MR image with T1-weighted (C) axial scan at the L5 pedicle level revealing a mass (arrow) in the left epidural space. The left L5 nerve root (open arrow) is between the mass (arrow) and the left L5 pedicle (star).
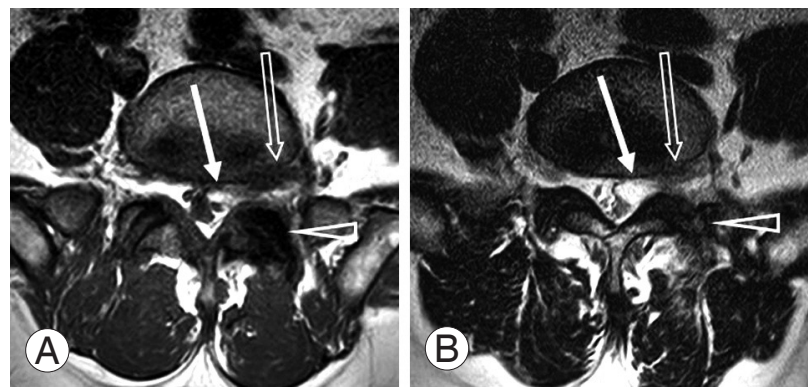

Fig. 2. T1-weighted (A) and T2-weighted (B) axial magnetic resonance images at the L5-S1 neural foramen level demonstrating the relationship between the left L5 nerve root (open arrow), the mass (arrow) and the left facet joint of L5-S1 (open arrowhead). The left facet joint of L5-S1 (open arrowhead) showing periarticular sclerosis and hypertrophy suggestive of degeneration. Perineural soft tissue around the left L5 nerve root (open arrow) showing reticular low signal intensity on T1-weighted image (A), and high signal intensity on T2-weighted image (B) suggestive of perineural edema.

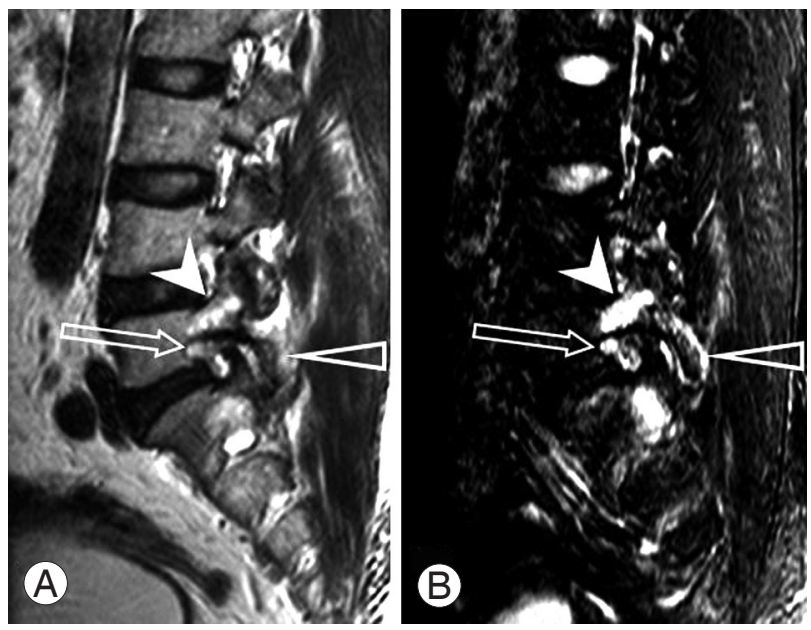

Fig. 3. Magnetic resonance images with T2-weighted sagittal scan (A) and fat suppressed T2-weighted sagittal scan (B) at the left L5 pedicle level showing degeneration of the left L5 pedicle (arrowhead) and left facet joint of L5-S1 (open arrowhead). Open arrow, left L5 nerve root. 


\section{Discussion}

There have been many reports on SEL. It is a condition of diffuse, abnormal proliferation of normal adipose tissue in the spinal canal of the thoracic and lumbar spine [3]. Most published reports discussed the etiologies of SEL, including obesity, chronic corticosteroids use and endocrinopathy. Symptoms of SEL vary with the location of SEL. They usually present with gradually progressing symptoms and are rarely associated with rapidly aggravated neurologic deficits. Although computed tomography (CT) can be used adjunctively, or to assess bony architecture, radiologic assessments are mostly conducted by using MRI. It is not easy to associate radicular symptoms with SEL. To the best of our knowledge, there exist only two case reports on solitary epidural lipoma in the literature $[4,5]$. One case describes a fall experienced by an obese (BMI $25 \mathrm{~kg} / \mathrm{m}^{2}$ ), elderly female with osteoporosis suspected of suffering Paget's disease of the spine who experienced coincidental unilateral lamina fracture at L5. The other case described spontaneous onset with long duration of symptom. Although myelography or CT can detect epidural mass compressing on the cauda equina, most diagnosis is made intraoperatively, or confirmed pathologically. The best radiologic assessment is based on MR imaging. SEL showed typical images, "Y" sign in the lumbosacral area, which is a Y shaped dural sac circumferentially compressed by excessive deposition of epidural fat, being hyperintense on T1WI and of intermediate intensity on T2WI, excluding epidural hematoma and excessive adipose tissue posterior to the spinal cord in the thoracic spine [3]. Sagittal MR images show the longitudinal extent of the epidural fat. However, in solitary epidural

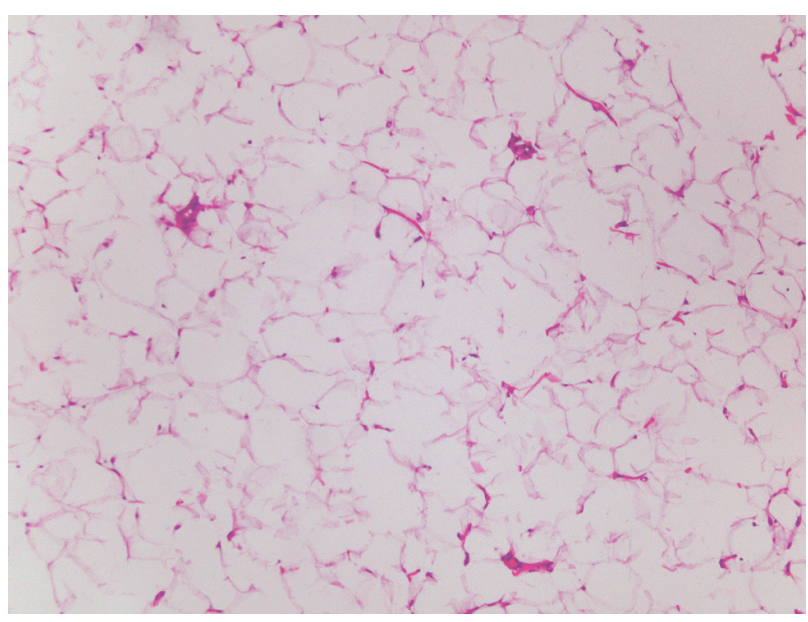

Fig. 4. Microscopic findings confirmed mature adipose tissue with large adipocytes $(\mathrm{H} \& \mathrm{E}, \times 100)$. lipoma, on MRI, both cases exhibited high signal solitary mass compressing cauda equina on T1WI without excessive proliferation of epidural adipose tissue. Besides this finding, our patient experienced severe arthritis of the posterior facet and edema of the pedicle on the symptomatic side. These findings represent lipoma on TI weighted images of MRI. Management of SEL depends on the severity of symptom and extent of neurologic deficit. There exist a few case reports that discussed clinical improvement following corticosteroid withdrawal or reduction and weight reduction, and the initial intervention for idiopathic epidural lipomatosis includes weight loss and exercise [6]. However, we have to be aware that urgent decompression may be needed in patients with progressive weakness of the extremities or bladder dysfunction [7]. However, two previously reported cases on patients with epidural lipoma were managed with surgical intervention due to intractable pain, inability to walk and neurologic deficit $[5,8]$. In our patient, the initial symptom was not relieved by conservative treatment, including physiotherapy and opioid analgesia, and he declined surgical intervention. Through SNRB, his symptoms were partially relieved and remained stable for 3 months. Eventually, severe pain and neurologic deficit recurred. Subsequent symptomatic exacerbation was not relieved by SNRB and finally he underwent decompressive surgery. SNRB can relieve severe symptoms caused by epidural lipoma-induced radiculopathy for a brief period, but it remains difficult to sustain. Disc or facet joint degeneration is thought to cause abnormal movement between spinal segments [9]. Kong et al. [10] reported that translational motion increased as facet joint degeneration increased. Although it remains unclear whether unilateral arthritis of the facet joint on the side of epidural lipoma exacerbated symptoms in our patient, we think that instability due to facet arthritis may make nerve root more vulnerable to compression due to lipoma, and instability may be a causative factor for abrupt symptom onset. Because solitary epidural lipoma is exceedingly rare and epidural lipoma is isosignal to normal epidural fat on MR images, it remains challenging to make an accurate diagnosis and therefore appropriate treatment may be delayed. For our patient, we had to take solitary epidural lipoma into consideration as a possible cause of lumbar radiculopathy. We recommend that solitary epidural lipoma causing neurologic deficit be excised at the time of diagnosis. This case report focused on solitary epidural lipoma associated with degenerative arthritis of ipsilateral posterior facet joints. 


\section{REFERENCES}

1. Badami JP, Hinck VC. Symptomatic deposition of epidural fat in a morbidly obese woman. AJNR Am J Neuroradiol 1982;3:664-5.

2. Haddad SF, Hitchon PW, Godersky JC. Idiopathic and glucocorticoid-induced spinal epidural lipomatosis. J Neurosurg 1991;74:38-42.

3. Borré DG, Borré GE, Aude F, Palmieri GN. Lumbosacral epidural lipomatosis: MRI grading. Eur Radiol 2003;13:1709-21.

4. Schizas C, Ballesteros C, Roy P. Cauda equina compression after trauma: an unusual presentation of spinal epidural lipoma. Spine (Phila Pa 1976) 2003;28:E14851.

5. Zevgaridis D, Nanassis K, Zaramboukas T. Lumbar nerve root compression due to extradural, intraforaminal lipoma. An underdiagnosed entity? J Neurosurg Spine 2008;9:408-10.

6. Beges C, Rousselin B, Chevrot A, et al. Epidural lipo- matosis. Interest of magnetic resonance imaging in a weight-reduction treated case. Spine (Phila Pa 1976) 1994;19:251-4.

7. López-González A, Resurrección Giner M. Idiopathic spinal epidural lipomatosis: urgent decompression in an atypical case. Eur Spine J 2008; 17 Suppl 2:S225-7.

8. Geers C, Lecouvet FE, Behets C, Malghem J, Cosnard G, Lengelé BG. Polygonal deformation of the dural sac in lumbar epidural lipomatosis: anatomic explanation by the presence of meningovertebral ligaments. AJNR Am J Neuroradiol 2003;24:1276-82.

9. Fujiwara A, Tamai K, An HS, et al. The relationship between disc degeneration, facet joint osteoarthritis, and stability of the degenerative lumbar spine. J Spinal Disord 2000;13:444-50.

10. Kong MH, Morishita $\mathrm{Y}, \mathrm{He} \mathrm{W}$, et al. Lumbar segmental mobility according to the grade of the disc, the facet joint, the muscle, and the ligament pathology by using kinetic magnetic resonance imaging. Spine (Phila Pa 1976) 2009;34:2537-44. 